\title{
Semidefinite Relaxation and Randomization for Dynamic Cell Association in Heterogeneous Networks
}

\author{
Steven Corroy and Rudolf Mathar \\ Institute for Theoretical Information Technology \\ RWTH Aachen University \\ Aachen, Germany \\ \{corroy, mathar\}@ti.rwth-aachen.de
}

\begin{abstract}
In this paper, we consider a heterogeneous network with one macro node and one pico node. We are concerned with the problem of associating users in the macro cell, to either the macro node or the pico node, in order to maximize the sum rate in the downlink. We formulate a new theoretical framework to study this problem and derive an upper bound on the achievable sum rate using semidefinite relaxation. Furthermore, we propose a randomized heuristic to produce a feasible solution, and most importantly, give an analytic guarantee on its performance. Independently of the problem data, we can ensure a worst case performance for the randomization method. In practice, this guarantee is as good as the standard best SNR heuristic typically used in 3GPP LTE networks.
\end{abstract}

\section{INTRODUCTION}

The main bottleneck of current mobile communication networks is the lack of available frequency resources. To tackle this problem, heterogeneous networks (HetNets) [1] are seen as a very promising approach. Inside a macro cell, with a macro node using a high transmit power, the idea is to use one or more pico nodes (using a lower transmit power), in areas where user equipments (UEs) are highly concentrated, to serve them using the same frequency resources as the macro node. This leads to a more efficient use of the available bandwidth and to higher data rates.

One of the key problems in HetNets is how to associate UEs to either the macro node or the pico nodes. The standard approach to this question, in 3GPP Long Term Evolution (LTE) networks, is the so called best SNR heuristic, which consists in associating a UE to the node providing the best signal-to-noise ratio (SNR). The main advantage of this approach is that it ensures a good signal-to-interference-plus-noise ratio (SINR) for each UE. The disadvantage is that, since the macro node uses a higher transmit power than the pico nodes, UEs tend to be mainly associated to the macro node [2], which leads to a load imbalance and a suboptimal sharing of the bandwidth.

In this paper, we want to derive a dynamic cell association scheme, which maximizes the sum rate for the downlink and analyze the trade-off between load balancing and SINR.

In general, the problem of load balancing in cell association has been studied for 3G networks. In [3], the authors propose a centralized approach to the cell association problem and

This work was partly supported by the UMIC research cluster of the RWTH Aachen University. consider a scenario in which each node serves a single UE at each time slot. In [4] and [5], the authors directly tackle the balancing problem by assuming a proportional fair scheduler. They derive the optimal cell association by solving a sequence of Boolean linear programs. Among others, the present work differs from these contributions because 1) we consider a HetNet scenario with a large imbalance between the transmit powers of macro node and pico nodes, 2) we model the specific trade-off between interference and load balancing and 3) we use convex optimization methods to derive an upper bound on the maximum sum rate.

More specifically, in [6] and [7], we have proposed a heuristic, which works very well in practice. Although it is very efficient when the channel gains between transmitter and receiver are more or less proportional to the distance between them, it may underperform in case of strong fading or obstacles presence. The main novelty of the present paper is 1) a more elegant theoretical framework, taking into account any kind of channel gains distribution, 2) a new heuristic based on randomization techniques and 3) we can guarantee the performance of this heuristic independently of the problem data, i.e., no matter how strong the channel fading is, our method always performs at least as good as this guarantee.

The remainder of the present paper is organized as follows. In Section II, we detail our theoretical framework and present the optimization problem to be solved. In Section III, we first give an upper bound on the achievable sum rate using semidefinite relaxation and then propose a randomized heuristic to produce a feasible cell association. In Section IV, we develop analytical bounds on the performance of this heuristic, in order to guarantee a minimum sum rate for any problem data set. In Section $\mathrm{V}$, we present numerical results to validate our work and finally Section VI concludes the present paper.

\section{System Model and Optimization Problem}

We consider downlink communication in a simple HetNet with one macro node, one pico node and $n$ UEs. Each UE should be associated to either the macro node or the pico node. This scenario is illustrated in Figure 1. Our goal is to find the association that maximizes the sum rate. Note that this metric is chosen for simplicity of exposition and the following 


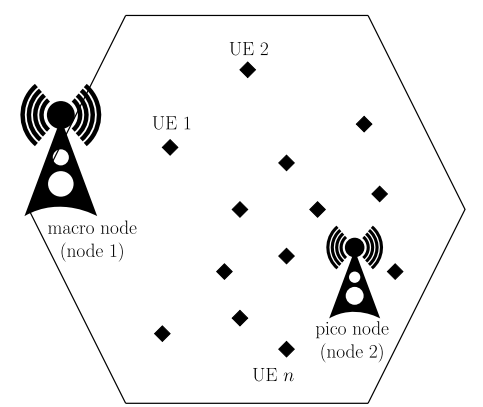

Fig. 1. Scenario with tightly coordinated macro and pico nodes.

approach can be used to maximize, a.o., a weighted sum rate, the minimum rate or the sum rate with rate constraints.

In the following we call the macro node, node 1 , and the pico node, node 2 . Node $i$ has a transmit power $\sigma_{i}$. Each UE $j$ receives a power $\hat{\sigma}_{i j}=g_{i j} \sigma_{i}$ from node $i$, where $g_{i j}$ denotes the channel gain between node $i$ and UE $j$. Furthermore we define $\sigma_{N}$ as the sum of the power of the noise on the channel and the power of the interference coming from outside the cell.

Each node utilizes the same frequency bandwidth $B$. In this paper we assume that each node always uses its complete available bandwidth and shares it equally among all its associated UEs. This models a Round-Robin scheduler. This has a very important consequence. Since we consider the downlink, if a node is active, it creates interference at all UEs that are not associated to it. This interference is treated as noise at the receivers.

In order to formulate a tractable optimization problem, we depict the following three cases.

1) All UEs are associated with node 1 . Only node 1 is active. The achieved sum rate $p_{1}$ is given by

$$
p_{1}=\frac{B}{n} \sum_{j=1}^{n} \log \left(1+\frac{\hat{\sigma}_{1 j}}{\sigma_{N}}\right) .
$$

2) All UEs are associated with node 2 . Only node 2 is active. The achieved sum rate $p_{2}$ is given by

$$
p_{2}=\frac{B}{n} \sum_{j=1}^{n} \log \left(1+\frac{\hat{\sigma}_{2 j}}{\sigma_{N}}\right) .
$$

3) At least one UE is associated with each node. Both node 1 and 2 are active. Define the rate vectors $\mathbf{r}_{1}$ and $\mathbf{r}_{2}$ as

$$
r_{i j}=B \log \left(1+\frac{\hat{\sigma}_{i j}}{\hat{\sigma}_{k j}+\sigma_{N}}\right), \quad k \neq i .
$$

Note that $r_{i j}$ represents the rate that UE $j$ would get from node $i$ if it would get the bandwidth $B$ allocated. The actual rate that UE $j$ gets, after that the bandwidth has been equally distributed among all UEs, is $r_{i j}$ divided by the number of UEs associated to node $i$.

To find the association that maximizes the sum rate, we can simply evaluate $p_{1}$ and $p_{2}$. We only have to formulate the sum rate maximization problem for the third case. We define an optimization variable $\mathbf{z}$, given by

$$
\begin{array}{ll}
z_{j}=1 & \text { if UE } j \text { is associated with node } 1, \\
z_{j}=-1 & \text { if UE } j \text { is associated with node } 2 .
\end{array}
$$

The problem of finding the association that maximizes the sum rate when at least one UE is associated with each node can be formulated as

$$
\begin{array}{ll}
\underset{\mathbf{z}}{\operatorname{maximize}} & \frac{\mathbf{r}_{1}^{\mathrm{T}}(\mathbf{1}+\mathbf{z})}{\mathbf{1}^{\mathrm{T}}(\mathbf{1}+\mathbf{z})}+\frac{\mathbf{r}_{2}^{\mathrm{T}}(\mathbf{1}-\mathbf{z})}{\mathbf{1}^{\mathrm{T}}(\mathbf{1}-\mathbf{z})} \\
\text { subject to } & z_{i} \in\{-1,1\}, i=1, \ldots, n,
\end{array}
$$

where 1 is a vector containing only ' 1 's. The domain $\mathcal{D}$ of the optimization problem is given by

$$
\mathcal{D}=\left\{\{-1,1\}^{n} \backslash\{-\mathbf{1}, \mathbf{1}\}\right\},
$$

the optimal value of (5) is denoted by $p^{\star}$ and the corresponding association by $\mathbf{z}^{\star}$.

Finally, to find the overall optimal cell association, we evaluate $\max \left(p_{1}, p_{2}, p^{\star}\right)$. If $p^{\star}$ is the maximum then $\mathbf{z}^{\star}$ is the optimal association, otherwise if $p_{i}$ is the maximum, all UEs are associated with node $i$. From now on we only consider problem (5).

\section{Semidefinite Relaxation and RAndomization}

The problem (5) can be reformulated as follows

$$
\begin{array}{ll}
\underset{\mathbf{z}}{\operatorname{maximize}} & \frac{\mathbf{z}^{\mathrm{T}} \mathbf{A}_{1} \mathbf{z}+\mathbf{b}_{1}^{\mathrm{T}} \mathbf{z}+c_{1}}{\mathbf{z}^{\mathrm{T}} \mathbf{A}_{2} \mathbf{z}+c_{2}} \\
\text { subject to } & z_{i} \in\{-1,1\}, i=1, \ldots, n,
\end{array}
$$

with $\mathbf{A}_{1} \triangleq-\left(\mathbf{r}_{1} \mathbf{1}^{\mathrm{T}}+\mathbf{r}_{2} \mathbf{1}^{\mathrm{T}}\right), \mathbf{b}_{1} \triangleq\left(n \mathbf{I}-\mathbf{1 1}^{\mathrm{T}}\right) \mathbf{r}_{1}+\left(\mathbf{1 1}^{\mathrm{T}}-\right.$ $n \mathbf{I}) \mathbf{r}_{2}, c_{1} \triangleq n\left(\mathbf{r}_{1}^{\mathrm{T}} \mathbf{1}+\mathbf{r}_{2}^{\mathrm{T}} \mathbf{1}\right), \mathbf{A}_{2} \triangleq-\mathbf{1 1}^{\mathrm{T}}$ and $c_{2} \triangleq n^{2}$.

Next it can easily be shown that the problem (7) is equivalent to solving the following problem

$$
\begin{array}{ll}
\underset{\mathbf{z}, t}{\operatorname{maximize}} & \frac{\mathbf{z}^{\mathrm{T}} \mathbf{A}_{1} \mathbf{z}+t \mathbf{b}_{1}^{\mathrm{T}} \mathbf{z}+t^{2} c_{1}}{\mathbf{z}^{\mathrm{T}} \mathbf{A}_{2} \mathbf{z}+t^{2} c_{2}} \\
\text { subject to } & z_{i} \in\{-1,1\}, i=1, \ldots, n, \\
& t \in\{-1,1\} .
\end{array}
$$

Indeed if $t=1$ then (8) is exactly (7). If $t=-1$ and $\mathbf{z}^{\star}$ is the solution of (8) then $-\mathbf{z}^{\star}$ is the solution of (7).

Finally we introduce a variable $\mathbf{x}=\left[\mathbf{z}^{\mathrm{T}} t\right]^{\mathrm{T}}$. The problem (8) can be rewritten as

$$
\begin{array}{ll}
\underset{\mathbf{x}}{\operatorname{maximize}} & \frac{\mathbf{x}^{\mathrm{T}} \mathbf{A} \mathbf{x}}{\mathbf{x}^{\mathrm{T}} \mathbf{B} \mathbf{x}} \\
\text { subject to } & x_{i} \in\{-1,1\}, i=1, \ldots, n+1,
\end{array}
$$

with

$$
\mathbf{A} \triangleq\left[\begin{array}{cc}
\mathbf{A}_{1} & (1 / 2) \mathbf{b}_{1} \\
(1 / 2) \mathbf{b}_{1}^{\mathrm{T}} & c_{1}
\end{array}\right], \quad \mathbf{B} \triangleq\left[\begin{array}{cc}
\mathbf{A}_{2} & \mathbf{0} \\
\mathbf{0}^{\mathrm{T}} & c_{2}
\end{array}\right],
$$

where $\mathbf{0}$ is a vector containing only ' 0 's. We denote by $q^{\star}$ the optimal value of (9). The domain $\mathcal{D}_{q}$ of (9) is

$$
\mathcal{D}_{q}=\left\{\{-1,1\}^{n+1} \backslash\left\{\mathbf{x} \mid x_{1}=\cdots=x_{n}= \pm 1\right\}\right\} .
$$

This problem is a hard combinatorial problem. Apart from the binary constraint, one of the main difficulties is that $\mathbf{A}$ 
and $\mathbf{B}$ are not definite. However, it is important to keep the following Lemma in mind.

Lemma 1. For all $\mathrm{x} \in\{-1,1\}^{n+1}$, it holds

$$
\mathbf{x}^{\mathrm{T}} \mathbf{A} \mathbf{x}>0, \quad \mathbf{x}^{\mathrm{T}} \mathbf{B} \mathbf{x}>0 \text {. }
$$

Proof:

$$
\begin{aligned}
\mathbf{x}^{\mathrm{T}} \mathbf{A} \mathbf{x}= & \mathbf{z}^{\mathrm{T}} \mathbf{A}_{1} \mathbf{z}+t \mathbf{b}_{1}^{\mathrm{T}} \mathbf{z}+t^{2} c_{1} \\
= & t^{2}\left(\mathbf{r}_{1}^{\mathrm{T}}(\mathbf{1}+\mathbf{z} / t) \mathbf{1}^{\mathrm{T}}(\mathbf{1}-\mathbf{z} / t)+\right. \\
& \left.\mathbf{r}_{2}^{\mathrm{T}}(\mathbf{1}-\mathbf{z} / t) \mathbf{1}^{\mathrm{T}}(\mathbf{1}+\mathbf{z} / t)\right) .
\end{aligned}
$$

Since $\mathbf{r}_{1}$ and $\mathbf{r}_{2}$ are strictly positive rate vectors and $\mathbf{z} / t \in$ $\{-1,1\}^{n}$, the rhs is positive. Now since at least one entry of $\mathbf{z}$ is 1 and at least one is -1 , both $\mathbf{1}+\mathbf{z} / t$ and $\mathbf{1}-\mathbf{z} / t$ have at least one strictly positive entry and $\mathrm{x}^{\mathrm{T}} \mathbf{A x}$ must be strictly positive. Similarly

$$
\mathbf{x}^{\mathrm{T}} \mathbf{B} \mathbf{x}=n^{2}-\mathbf{z}^{\mathrm{T}} \mathbf{1} \mathbf{1}^{\mathrm{T}} \mathbf{z} .
$$

Since at least one entry of $\mathbf{z}$ is 1 and at least one is -1 , $\mathbf{z}^{\mathrm{T}} \mathbf{1} \mathbf{1}^{\mathrm{T}} \mathbf{z}<n^{2}$.

In the next sections, we find an upper bound on the optimal value of problem (9), then propose a randomization algorithm producing a feasible solution and finally give a guarantee on the performance of this heuristic.

\section{A. Sum Rate Upper Bound using Semidefinite Relaxation}

Since the problem (9) is hard to solve, we will first try to find a good upper bound on $q^{\star}$. In order to do that, we proceed to the following change of variable $\mathbf{X}=\mathbf{x x}^{\mathrm{T}}$. By noting that $\mathbf{X}=\mathbf{x} \mathbf{x}^{\mathrm{T}}$ is equivalent to saying that $\mathbf{X}$ has rank one and is positive semidefinite, the problem (9) is equivalent to

$$
\begin{array}{cl}
\underset{\mathbf{X}}{\operatorname{maximize}} & \frac{\operatorname{tr}(\mathbf{A X})}{\operatorname{tr}(\mathbf{B X})} \\
\text { subject to } & \operatorname{diag}(\mathbf{X})=\mathbf{1}, \\
& \mathbf{X} \succcurlyeq \mathbf{0}, \quad \operatorname{rank}(\mathbf{X})=1, \\
& \sum_{i=1}^{n} \sum_{j=1}^{n} X_{i j} \leq n^{2}-2(n-1),
\end{array}
$$

where the last constraint makes the domain $\mathcal{D}_{q}$ of problem (9) explicit, in the form of a sum over the components of $\mathbf{X}$.

To make problem (9) tractable and to upper bound $q^{\star}$, we relax (11) by dropping the rank constraint (which is nonconvex) and solve

$$
\begin{array}{ll}
\underset{\mathbf{X}}{\operatorname{maximize}} & \frac{\operatorname{tr}(\mathbf{A X})}{\operatorname{tr}(\mathbf{B X})} \\
\text { subject to } & \operatorname{diag}(\mathbf{X})=\mathbf{1} \\
& \mathbf{X} \succcurlyeq \mathbf{0}, \sum_{i=1}^{n} \sum_{j=1}^{n} X_{i j} \leq n^{2}-2(n-1) .
\end{array}
$$

The solution of (12) is denoted by $\mathbf{X}^{\star}$ and the optimal value by $s^{\star}$.

This problem is a quasiconcave problem [8] since all its constraints are convex and its objective function is quasiconcave. This problem can be solved efficiently, using, e.g., the bisection method, with free open source software packages like, e.g., CVX [9]. Since (12) is a relaxation of (9), it holds

$$
q^{\star} \leq s^{\star}=\frac{\operatorname{tr}\left(\mathbf{A} \mathbf{X}^{\star}\right)}{\operatorname{tr}\left(\mathbf{B X}^{\star}\right)}
$$

Next we propose a heuristic to produce a feasible solution for the problem (9).

\section{B. Randomization Heuristic}

The principles of the randomization method to solve combinatorial problems have been introduced in [10], [11] and [12].

The proposed algorithm in our case, goes as follows.

1) Solve (12) and find $\mathbf{X}^{\star}$.

2) Generate a random vector $\mathbf{y} \sim \mathcal{N}\left(\mathbf{0}, \mathbf{X}^{\star}\right)$.

3) Evaluate $\mathbf{x}=\operatorname{sgn}(\mathbf{y})$, where $\operatorname{sgn}$ is the sign function. If $\mathbf{x} \in \mathcal{D}_{q}$, then it is feasible for (9), else it is ignored.

4) Take $\mathbf{z}=x_{n+1}\left[\begin{array}{lll}x_{1} & \ldots & x_{n}\end{array}\right]^{\mathrm{T}}$. The vector $\mathbf{x}$ is a feasible solution of (5).

5) Repeat from step 2) and always keep the association providing the best sum rate.

The number of iterations does not have to be large. Mainly we are interested in getting a good solution (in contrast to optimal) and this is achieved relatively quickly. We call $r^{\star}$ the sum rate obtained by the randomization algorithm. On average, this method achieves an average sum rate of $\mathbb{E}_{\mathbf{x}}\left[\mathbf{x}^{\mathrm{T}} \mathbf{A} \mathbf{x} / \mathbf{x}^{\mathrm{T}} \mathbf{B} \mathbf{x}\right]$. Since we only keep the best realization of the randomization algorithm, with overwhelming probability it holds that $r^{\star} \geq \mathbb{E}_{\mathbf{x}}\left[\mathbf{x}^{\mathrm{T}} \mathbf{A} \mathbf{x} / \mathbf{x}^{\mathrm{T}} \mathbf{B} \mathbf{x}\right]$. Finally the association produced by the randomization heuristic is not necessary optimal and we have

$$
\mathbb{E}_{\mathbf{x}}\left[\frac{\mathbf{x}^{\mathrm{T}} \mathbf{A} \mathbf{x}}{\mathbf{x}^{\mathrm{T}} \mathbf{B} \mathbf{x}}\right] \leq r^{\star} \leq q^{\star} \leq s^{\star}=\frac{\operatorname{tr}\left(\mathbf{A} \mathbf{X}^{\star}\right)}{\operatorname{tr}\left(\mathbf{B X}^{\star}\right)}
$$

In the next section, we present results showing how tight these bounds can be.

\section{Performance Guarantee}

In this section we want to characterize the ratio $r^{\star} / s^{\star}$. If we can show that $r^{\star} / s^{\star} \geq \alpha$, then it holds that the randomization method always provides a sum rate greater than or equal to $\alpha q^{\star}$. If $\alpha$ is independent of $\mathbf{A}$ and $\mathbf{B}$, we can guarantee a sum rate of $\alpha q^{\star}$ for any possible channel gain distribution. In order to obtain a guarantee on the performance of the randomization method, we have to evaluate the expression $\mathbb{E}_{\mathbf{x}}\left[\mathbf{x}^{\mathrm{T}} \mathbf{A x} / \mathbf{x}^{\mathrm{T}} \mathbf{B} \mathbf{x}\right]$ and compare it to $\operatorname{tr}\left(\mathbf{A} \mathbf{X}^{\star}\right) / \operatorname{tr}\left(\mathbf{B} \mathbf{X}^{\star}\right)$.

Definition 1. The joint moment generating function of the two functions $\mathbf{x}^{\mathrm{T}} \mathbf{A} \mathbf{x}$ and $\mathbf{x}^{\mathrm{T}} \mathbf{B} \mathbf{x}$ is given by

$$
M\left(t_{1}, t_{2}\right)=\mathbb{E}_{\mathbf{x}}\left[\exp \left(t_{1} \mathbf{x}^{\mathrm{T}} \mathbf{A} \mathbf{x}+t_{2} \mathbf{x}^{\mathrm{T}} \mathbf{B} \mathbf{x}\right)\right] .
$$

Lemma 2.

$$
\mathbb{E}_{\mathbf{x}}\left[\frac{\mathbf{x}^{\mathrm{T}} \mathbf{A} \mathbf{x}}{\mathbf{x}^{\mathrm{T}} \mathbf{B} \mathbf{x}}\right]=\left.\int_{-\infty}^{0} \frac{\partial M\left(t_{1}, t_{2}\right)}{\partial t_{1}}\right|_{t_{1}=0} d t_{2} .
$$




$$
\begin{aligned}
& \text { Proof: } \\
& \begin{aligned}
\left.\int_{-\infty}^{0} \frac{\partial M\left(t_{1}, t_{2}\right)}{\partial t_{1}}\right|_{t_{1}=0} d t_{2} \\
\quad=\left.\int_{-\infty}^{0} \frac{\partial \mathbb{E}_{\mathbf{x}}\left[\exp \left(t_{1} \mathbf{x}^{\mathrm{T}} \mathbf{A} \mathbf{x}+t_{2} \mathbf{x}^{\mathrm{T}} \mathbf{B} \mathbf{x}\right)\right]}{\partial t_{1}}\right|_{t_{1}=0} d t_{2} \\
\quad=\mathbb{E}_{\mathbf{x}}\left[\left.\int_{-\infty}^{0} \frac{\partial \exp \left(t_{1} \mathbf{x}^{\mathrm{T}} \mathbf{A} \mathbf{x}+t_{2} \mathbf{x}^{\mathrm{T}} \mathbf{B} \mathbf{x}\right)}{\partial t_{1}}\right|_{t_{1}=0} d t_{2}\right] \\
\quad=\mathbb{E}_{\mathbf{x}}\left[\int_{-\infty}^{0} \mathbf{x}^{\mathrm{T}} \mathbf{A x} \exp \left(t_{2} \mathbf{x}^{\mathrm{T}} \mathbf{B} \mathbf{x}\right) d t_{2}\right] \\
\quad=\mathbb{E}_{\mathbf{x}}\left[\frac{\mathbf{x}^{\mathrm{T}} \mathbf{A} \mathbf{x}}{\mathbf{x}^{\mathrm{T}} \mathbf{B} \mathbf{x}}\right] .
\end{aligned}
\end{aligned}
$$

\section{Proposition 1.}

$$
\mathbb{E}_{\mathbf{x}}\left[\frac{\mathbf{x}^{\mathrm{T}} \mathbf{A} \mathbf{x}}{\mathbf{x}^{\mathrm{T}} \mathbf{B} \mathbf{x}}\right] \simeq \frac{\mathbb{E}_{\mathbf{x}}\left[\mathbf{x}^{\mathrm{T}} \mathbf{A} \mathbf{x}\right]}{\mathbb{E}_{\mathbf{x}}\left[\mathbf{x}^{\mathrm{T}} \mathbf{B} \mathbf{x}\right]}
$$

Proof: The proof follows the one of [13] originally for B positive definite. It is however simplified, more detailed and adapted to our present scenario. It is mainly based on Laplace approximation. We first decompose the rhs of Lemma 2 as follows

$$
\begin{gathered}
\left.\int_{-\infty}^{0} \frac{\partial \mathbb{E}_{\mathbf{x}}\left[\exp \left(t_{1} \mathbf{x}^{\mathrm{T}} \mathbf{A} \mathbf{x}+t_{2} \mathbf{x}^{\mathrm{T}} \mathbf{B} \mathbf{x}\right)\right]}{\partial t_{1}}\right|_{t_{1}=0} d t_{2} \\
=\int_{-\infty}^{0} \mathbb{E}_{\mathbf{x}}\left[\mathbf{x}^{\mathrm{T}} \mathbf{A} \mathbf{x} \exp \left(t_{2} \mathbf{x}^{\mathrm{T}} \mathbf{B} \mathbf{x}\right)\right] d t_{2} \\
=\int_{-\infty}^{0} f_{1}\left(t_{2}\right) \exp \left(f_{2}\left(t_{2}\right)\right) d t_{2},
\end{gathered}
$$

with

$$
f_{1}\left(t_{2}\right)=\frac{\mathbb{E}_{\mathbf{x}}\left[\mathbf{x}^{\mathrm{T}} \mathbf{A} \mathbf{x} \exp \left(t_{2} \mathbf{x}^{\mathrm{T}} \mathbf{B} \mathbf{x}\right)\right]}{\mathbb{E}_{\mathbf{x}}\left[\exp \left(t_{2} \mathbf{x}^{\mathrm{T}} \mathbf{B} \mathbf{x}\right)\right]},
$$

and

$$
f_{2}\left(t_{2}\right)=\log \left(\mathbb{E}_{\mathbf{x}}\left[\exp \left(t_{2} \mathbf{x}^{\mathrm{T}} \mathbf{B} \mathbf{x}\right)\right]\right) .
$$

Now we will approximate this integral using Laplace method. Since $\mathbf{x}^{\mathrm{T}} \mathbf{B x}>0$ on the feasible set (see Lemma 1) and the log function is strictly increasing, then $f_{2}\left(t_{2}\right)$ attains its maximum at the boundary of the integral, i.e., at $t_{2, \max }=0$. The key here is to understand that the points $t_{2}$ around $t_{2, \max }$ are going to make the most significant contribution to the integral. The Laplace method consists in replacing $f_{1}\left(t_{2}\right)$ by $f_{1}\left(t_{2, \max }\right)$ and making a first order Taylor approximation of $f_{2}$ at $t_{2, \max }$, namely

$$
f_{2}\left(t_{2}\right) \simeq \log (1)+t_{2} \mathbb{E}_{\mathbf{x}}\left[\mathbf{x}^{\mathrm{T}} \mathbf{B} \mathbf{x}\right] .
$$

It follows that

$$
\int_{-\infty}^{0} f_{1}\left(t_{2}\right) \exp \left(f_{2}\left(t_{2}\right)\right) d t_{2}
$$

$$
\begin{aligned}
& \simeq \int_{-\infty}^{0} f_{1}\left(t_{2, \max }\right) \exp \left(t_{2} \mathbb{E}_{\mathbf{x}}\left[\mathbf{x}^{\mathrm{T}} \mathbf{B} \mathbf{x}\right]\right) d t_{2} \\
& =\int_{-\infty}^{0} \mathbb{E}_{\mathbf{x}}\left[\mathbf{x}^{\mathrm{T}} \mathbf{A} \mathbf{x}\right] \exp \left(t_{2} \mathbb{E}_{\mathbf{x}}\left[\mathbf{x}^{\mathrm{T}} \mathbf{B} \mathbf{x}\right]\right) d t_{2} . \\
& =\frac{\mathbb{E}_{\mathbf{x}}\left[\mathbf{x}^{\mathrm{T}} \mathbf{A} \mathbf{x}\right]}{\mathbb{E}_{\mathbf{x}}\left[\mathbf{x}^{\mathrm{T}} \mathbf{B} \mathbf{x}\right]}
\end{aligned}
$$

Lemma 3.

$$
\mathbb{E}_{\mathbf{x}}\left[\frac{\mathbf{x}^{\mathrm{T}} \mathbf{A} \mathbf{x}}{\mathbf{x}^{\mathrm{T}} \mathbf{B} \mathbf{x}}\right] \geq \frac{\mathbb{E}_{\mathbf{x}}\left[\mathbf{x}^{\mathrm{T}} \mathbf{A} \mathbf{x}\right]}{\mathbb{E}_{\mathbf{x}}\left[\mathbf{x}^{\mathrm{T}} \mathbf{B} \mathbf{x}\right]}+\mathcal{O}\left(n^{-1}\right)
$$

Proof: This bound comes directly from the error induced by the approximation in Proposition (1). In [13], the author shows that if $\mathbb{E}_{\mathbf{x}}\left[\mathbf{x}^{\mathrm{T}} \mathbf{A} \mathbf{x}\right]=\mathcal{O}(n)$, the cumulants of $\mathbf{x}^{\mathrm{T}} \mathbf{B} \mathbf{x}$ and the joint cumulants of $\mathbf{x}^{\mathrm{T}} \mathbf{A} \mathbf{x}$ and $\mathbf{x}^{\mathrm{T}} \mathbf{B} \mathbf{x}$ are of order $n$, then the approximation error is of order $n^{-1}$.

Note that in the following, since we generate many $x$ 's at random and keep the best realization, we actually achieve a sum rate $r^{\star}$ greater than $\mathbb{E}_{\mathbf{x}}\left[\mathbf{x}^{\mathrm{T}} \mathbf{A x} / \mathbf{x}^{\mathrm{T}} \mathbf{B} \mathbf{x}\right]$, and we can neglect the term $\mathcal{O}\left(n^{-1}\right)$.

\section{Lemma 4.}

$$
\begin{aligned}
& \mathbb{E}_{\mathbf{x}}\left[\mathbf{x}^{\mathrm{T}} \mathbf{A} \mathbf{x}\right]=\frac{2}{\pi} \operatorname{tr}\left(\mathbf{A} \arcsin \left(\mathbf{X}^{\star}\right)\right), \\
& \mathbb{E}_{\mathbf{x}}\left[\mathbf{x}^{\mathrm{T}} \mathbf{B} \mathbf{x}\right]=\frac{2}{\pi} \operatorname{tr}\left(\mathbf{B} \arcsin \left(\mathbf{X}^{\star}\right)\right) .
\end{aligned}
$$

Proof: See [14] or [12], for a detailed proof.

We can now state one of the main results of this paper, which is a guarantee on the performance achieved by randomization.

\section{Proposition 2.}

$$
\frac{r^{\star}}{s^{\star}} \geq \frac{\operatorname{tr}\left(\mathbf{A} \arcsin \left(\mathbf{X}^{\star}\right)\right) \operatorname{tr}\left(\mathbf{B} \mathbf{X}^{\star}\right)}{\operatorname{tr}\left(\mathbf{B} \arcsin \left(\mathbf{X}^{\star}\right)\right) \operatorname{tr}\left(\mathbf{A} \mathbf{X}^{\star}\right)} .
$$

Proof: We simply plug Lemma 3 and 4 in (14).

As we will see, in practice, this bound is quite tight. In the following we present other bounds, independent of $\mathbf{A}, \mathbf{B}$ or $\mathbf{X}^{\star}$. These bounds will be looser but in a sense more general.

\section{Lemma 5.}

$$
\operatorname{tr}\left(\mathbf{A} \arcsin \left(\mathbf{X}^{\star}\right)\right) \geq \operatorname{tr}\left(\mathbf{A} \mathbf{X}^{\star}\right) .
$$

Proof: As shown in [11], it holds that

$$
\arcsin \left(\mathbf{X}^{\star}\right) \succcurlyeq \mathbf{X}^{\star} \text {. }
$$

It remains to evaluate the difference between the lhs and rhs.

$$
\begin{aligned}
\operatorname{tr}\left(\mathbf{A} \arcsin \left(\mathbf{X}^{\star}\right)\right) & -\operatorname{tr}\left(\mathbf{A} \mathbf{X}^{\star}\right) \\
= & \operatorname{tr}\left(\mathbf{A}\left(\arcsin \left(\mathbf{X}^{\star}\right)-\mathbf{X}^{\star}\right)\right) \\
\geq & \left.\lambda_{\min }\left(\arcsin \left(\mathbf{X}^{\star}\right)-\mathbf{X}^{\star}\right)\right) \operatorname{tr}(\mathbf{A})
\end{aligned}
$$

Since $\arcsin \left(\mathbf{X}^{\star}\right) \succcurlyeq \mathbf{X}^{\star}$, we have $\lambda_{\min }\left(\arcsin \left(\mathbf{X}^{\star}\right)-\mathbf{X}^{\star}\right) \geq 0$. Finally $\operatorname{tr}(\mathbf{A})=(n-1) \mathbf{1}^{\mathrm{T}}\left(\mathbf{r}_{1}+\mathbf{r}_{2}\right) \geq 0$.

Finally we give a guarantee on the performance of the randomization heuristic, which is independent of the problem data. 


\section{Proposition 3.}

$$
\frac{r^{\star}}{s^{\star}} \geq \frac{\operatorname{tr}\left(\mathbf{B X}^{\star}\right)}{\operatorname{tr}\left(\mathbf{B} \arcsin \left(\mathbf{X}^{\star}\right)\right)} .
$$

Proof: We simply use Lemma 5 in Proposition 2.

This bound, while less tight than the previous one, is very interesting since it does not depends on $\mathbf{A}$ and consequently on the rate vectors.

Corollary 1. For $i, j=1, \ldots, n$, it holds

$$
\frac{r^{\star}}{s^{\star}} \geq \frac{n^{2}-n-2 \sum_{i>j} X_{i j}^{\star}}{\frac{\pi}{2}\left(n^{2}-n\right)-2 \sum_{i>j} \arcsin \left(X_{i j}^{\star}\right)} .
$$

Proof: This is immediate by using the fact that $\mathbf{A}_{2}=$ $-\mathbf{1 1}^{\mathrm{T}}, c_{2}=n^{2}$ and $\operatorname{diag}\left(\mathbf{X}^{\star}\right)=\mathbf{1}$.

\section{Proposition 4.}

$$
\frac{r^{\star}}{s^{\star}} \geq \frac{4}{n\left(\frac{\pi}{2}-1\right)+\pi+2}
$$

Proof: Define

$$
\begin{gathered}
a_{1} \triangleq\left(\sum_{i>j} X_{i j}^{\star} \mid X_{i j}^{\star} \geq 0\right), \\
b_{1} \triangleq\left(\sum_{i>j} X_{i j}^{\star} \mid X_{i j}^{\star}<0\right), \\
a_{2} \triangleq\left(\sum_{i>j} \arcsin \left(X_{i j}^{\star}\right) \mid X_{i j}^{\star} \geq 0\right), \\
b_{2} \triangleq\left(\sum_{i>j} \arcsin \left(X_{i j}^{\star}\right) \mid X_{i j}^{\star}<0\right) .
\end{gathered}
$$

We decompose and bound the rhs of Corollary 1 as follows

$$
\begin{aligned}
\frac{r^{\star}}{s^{\star}} & \geq \frac{n^{2}-n-2\left(a_{1}+b_{1}\right)}{\frac{\pi}{2}\left(n^{2}-n\right)-2\left(a_{2}+b_{2}\right)} \\
& \geq \frac{n^{2}-n-2\left(a_{1}+b_{1}\right)}{\frac{\pi}{2}\left(n^{2}-n\right)-2\left(a_{1}+\frac{\pi}{2} b_{1}\right)} .
\end{aligned}
$$

Since from problem (12), it holds that

$$
\sum_{i=1}^{n} \sum_{j=1}^{n} X_{i j} \leq n^{2}-2(n-1),
$$

we have $a_{1} \leq(n-1)^{2}-(n-1)$ and $b_{1} \geq 2(n-1)$. It is not difficult to show that the last ratio above attains its minimum at the boundaries, i.e.,

$$
\frac{r^{\star}}{s^{\star}} \geq \frac{4}{n\left(\frac{\pi}{2}-1\right)+\pi+2}
$$

This bound is very useful since it is totally independent of the problem data. For any instance of the data, we can guarantee that randomization is no more suboptimal than this bound. Of course this bound goes to 0 when $n$ grows. But there are two important facts to note. First, this bound simply tends to indicate that randomization will perform relatively worse when the number of UEs is large. Second, we have to keep in mind that this guarantee is relative to an upper bound. If this upper bound would grow at the same speed as the guarantee decrease, we would have a constant absolute guarantee with respect to $n$, as shown in the next section.

\section{Numerical EVALUATION}

In this section we evaluate the performance of the randomization method with respect to the problem size and compared to the best SNR heuristic used in 3GPP LTE networks. The different cell associations are evaluated in a simplified radio network composed of one macro node with a Tx power of $40 \mathrm{~W}$ and one pico node with a Tx power of $1 \mathrm{~W}$. Each node operates with a $5 \mathrm{MHz}$ bandwidth. The pico node is randomly dropped within the macro cell area with a radius of $167 \mathrm{~m}$.

As proposed in [15], we calculate the average channel gain between the macro node and a user $j$ as $g_{1 j}=-(128.1+$ $\left.37.6 \log \left(d_{1 j}\right)\right)$, while the average channel gain between the pico node and a user $j$ is given by $g_{2 j}=-(140.7+$ $36.7 \log \left(d_{2 j}\right)$ ), where $d_{i j}$ denotes the distance between the user $j$ and the node $i$ in $\mathrm{km}$. The users are dropped in a hotspot of $40 \mathrm{~m}$ radius around the pico node with a probability of $2 / 3$. The following results are generated by averaging over 100 user drops.

In Figure 2, we plot the upper bound obtained by solving problem (11), the sum rate achieved by the randomization method, the best SNR heuristic and finally the guaranteed sum rate, attained according to Proposition (4). There are 10 UEs deployed and the noise and interference level goes from -160 to $-80 \mathrm{dBW}$. The first thing to observe is that the randomization heuristic clearly outperforms the best SNR heuristic for all noise levels, and the gap is extremely large when the noise level is low. Then we can compare the sum rate achieved by the randomization method to the upper bound and the guaranteed sum rate. The randomization method performs close to the upper bound in low and high SNR regime. However it is further away in the moderate SNR regime. From previous results in [6], we can say that the randomization method does not performs more poorly in the moderate SNR regime. By comparing the upper bound in [6] and the present one we can say that the present upper bound is simply less tight in this regime. The guaranteed sum rate is stable in high and moderate SNR regime and converges with the rate obtained using randomization in the low SNR regime. Finally, the most interesting result can be observed by comparing the best SNR heuristic and the guaranteed sum rate. The two curves are very close to each other, the best SNR heuristic being only slightly better. In other words, the randomization method is guaranteed, to perform at least as good as the best SNR heuristic, for any possible instance of problem data.

In Figure 3, we plot the distribution of pico users for different noise levels for the randomization method and the best SNR heuristic. Since the best SNR heuristic only depends on the received powers from the macro and pico nodes, its 


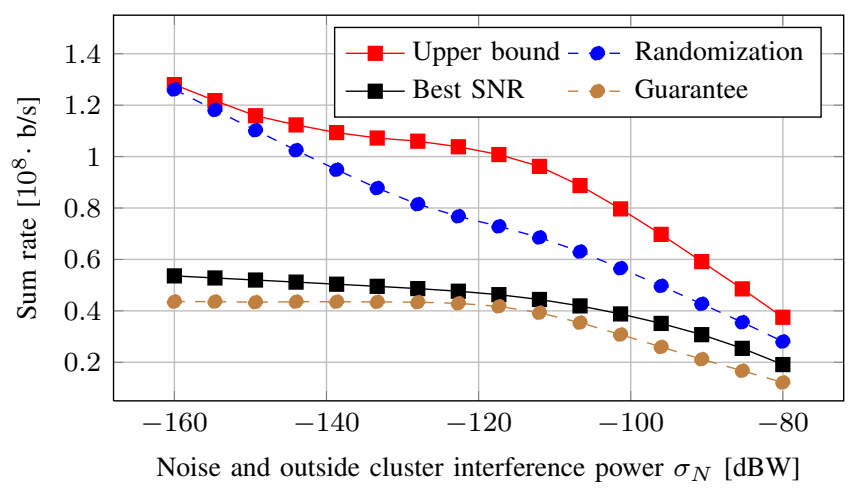

Fig. 2. Sum rate for different $\sigma_{N}$ values, number of UEs $=10$.

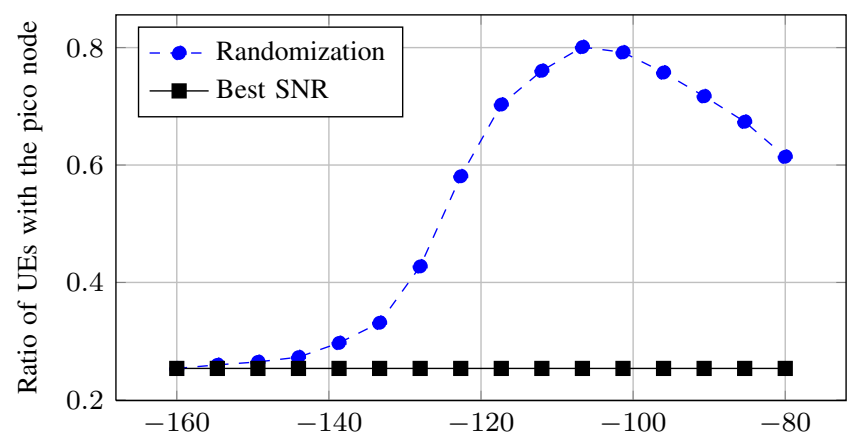

Noise and outside cluster interference power $\sigma_{N}[\mathrm{dBW}]$

Fig. 3. Ratio of UEs with the pico node, number of UEs $=10$.

cell association is always the same. The randomization uses the same pico users ratio as the best SNR heuristic, at a low noise level, increases to $80 \%$ in the moderate noise level and decreases again when the noise level is high. Interestingly, it is when the noise level is low that randomization outperforms the best SNR heuristic the most. In this case, the randomization chooses a completely different strategy, but in average, over all drops, the ratio of pico users is the same. It does not mean that the strategy is the same.

In Figure 4, we plot the sum rate with respect to the number of UEs in the macro cell when $\sigma_{N}=-128 \mathrm{dBW}$. The striking observation here is that the guaranteed sum rate is nearly constant and close to the sum rate achieved by the best SNR heuristic. From Proposition 4, we showed that the ratio $r^{\star} / s^{\star}$, was decreasing with $n$ and one could expect that the guaranteed rate would do the same. On the contrary, because the upper bound increases linearly with $n$, at around the same speed as the ratio in Proposition 4 decreases, then we can guarantee a constant sum rate independent of $n$. In conclusion, the randomization method perform in average better than the best SNR heuristic for any number of nodes, and is guaranteed to perform at least as good as the best SNR heuristic.

\section{CONCLUSION}

In this work, we have presented a new approach to the problem of cell association for the downlink of HetNets. We have proposed a semidefinite relaxation of the maximum sum rate problem and developed a randomized heuristic to find a

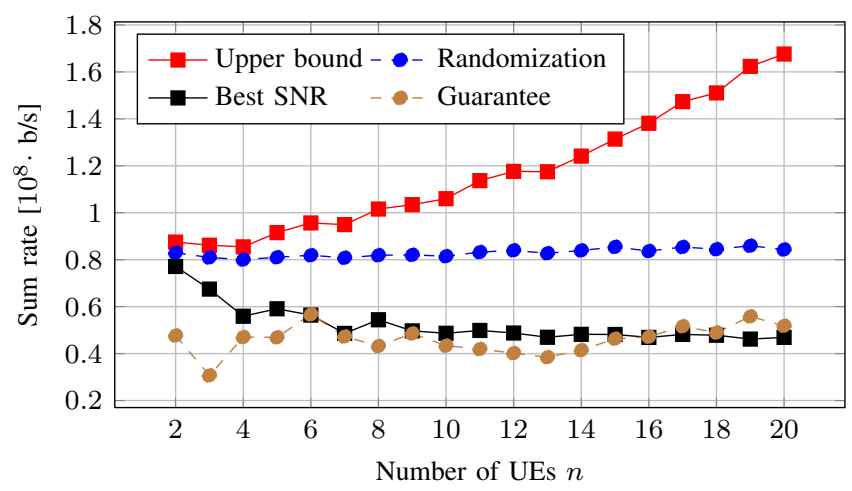

Fig. 4. Sum rate for different number of UEs, $\sigma_{N}=-128 \mathrm{dBW}$.

feasible association. Furthermore we have given an analytical guarantee on the performance of this heuristic, which does not depend on the problem data. Finally we have verified our results through numerical evaluations and shown that our approach outperforms the standard best SNR heuristic, while guaranteeing a similar worst-case sum rate. In future works we want to consider scenarios with several cells, as well as several pico nodes per cell.

\section{REFERENCES}

[1] S. Landström, A. Furuskär, K. Johansson, L. Falconetti, and F. Kronestedt, "Heterogeneous networks-increasing cellular capacity," Ericsson Review, pp. 4-9, 2011.

[2] NTT-DOCOMO, "Performance of eICIC with Control Channel Coverage Limitation," 3GPP TSG RAN WG1 Meeting 61, R1-103264.

[3] S. Das, H. Viswanathan, and G. Rittenhouse, "Dynamic load balancing through coordinated scheduling in packet data systems," in Proc. IEEE INFOCOM 2003, Mar. 2003, pp. 786 - 796 vol.1.

[4] T. Bu, L. Li, and R. Ramjee, "Generalized proportional fair scheduling in third generation wireless data networks," in Proc. IEEE INFOCOM 2006, Apr. 2006.

[5] K. Son, S. Chong, and G. Veciana, "Dynamic association for load balancing and interference avoidance in multi-cell networks," IEEE Trans. on Wireless Commun., vol. 8, pp. 3566 - 3576, Jul. 2009.

[6] S. Corroy, L. Falconetti, and R. Mathar, "Cell association in small heterogeneous networks: Downlink sum rate and min rate maximization," in To be presented at IEEE Wireless Communications and Networking Conference (WCNC 2012), Paris, France, Apr. 2012.

[7] _ - "Dynamic cell association for downlink sum rate maximization in multi-cell heterogeneous networks," in To be presented at IEEE International Conference on Communications (ICC 2012), Ottawa, Canada, Jun. 2012.

[8] S. Boyd and L. Vandenberghe, Convex optimization. Cambridge university press, 2008 (sixth printing).

[9] M. Grant and S. Boyd. (2009) CVX: Matlab software for disciplined convex programming (web page and software). [Online]. Available: http://stanford.edu/ boyd/cvx

[10] M. Goemans and D. Williamson, "Improved approximation algorithms for maximum cut and satisfiability problems using semidefinite programming," Journal of the ACM (JACM), vol. 42, no. 6, pp. 1115-1145, 1995.

[11] Y. Nesterov, "Semidefinite relaxation and nonconvex quadratic optimization," Optimization methods and software, vol. 9, no. 1-3, pp. 141-160, 1998.

[12] D. Bertsimas and Y. Ye, "Semidefinite relaxations, multivariate normal distributions, and order statistics," Handbook of Combinatorial Optimization, vol. 3, pp. 1-19, 1998.

[13] O. Lieberman, "A laplace approximation to the moments of a ratio of quadratic forms," Biometrika, vol. 81, no. 4, p. 681, 1994.

[14] W. F. Sheppard, "On the application of the theory of error to cases of normal distribution and normal correlation," Philosophical Transactions of the Royal Society of London, vol. 192, pp. 101-167, 1899.

[15] "3GPP TR36.814, Further advancements for E-UTRA physical layer aspects." 